\title{
ANATOMIA DO TUBO DIGESTÓRIO DA ESPÉCIE Acestrorhynchus lacustris (OSTARYOHISI, CHARACIFORMES) LÜTKEN, 1875 DO RIO DE ONDAS, OESTE DA BAHIA, BRASIL
}

\author{
Walter França Guedes ${ }^{1}$ \\ Rosana Marques Silva ${ }^{1}$ \\ Michel Castro Moreira ${ }^{2}$ \\ Lourdes Marina Bezerra Pessoa ${ }^{3}$ \\ Elis Regina Rodrigues de Souza Castro ${ }^{1}$
}

\begin{abstract}
RESUMO
O presente estudo visa descrever e mensurar o tubo digestório da espécie Acestrorhynchus lacustris. Foram utilizados 10 exemplares da espécie, coletados em diferentes trechos da bacia hidrográfica do rio de Ondas, localizada no Oeste do Estado da Bahia, ecorregião aquática do rio São Francisco. Foram realizadas mensuração e pesagem dos peixes. A cavidade bucofaríngea e os órgãos digestórios pós-faríngeos: esôfago, estômago, cecos pilóricos e intestino foram analisados, descritos e mensurados. Para definição do tamanho dos órgãos da espécie, independente do tamanho do exemplar, foi calculado o comprimento relativo dos órgãos, exceto dos cecos pilóricos. A espécie A. lacustris apresenta boca terminal, fenda bucal e cavidade bucofaringea amplas, dentes cônicos e caninos; esôfago curto, musculoso e distensível; estômago alongado, musculoso, do tipo cecal, com formato de "Y"; sete a 15 cecos pilóricos que aparecem como evaginações no intestino; e intestino curto, musculoso e bastante espesso. Os dados obtidos demonstram que o tamanho dos órgãos que compõe o tubo digestório da espécie não está relacionado ao tamanho do exemplar. O estudo das características anatômicas do tubo digestório ampliou o conhecimento biológico e ecológico da espécie estudada, auxiliando na compreensão do desempenho desta espécie em ecossistemas naturais e na elaboração de propostas para a preservação ou manejo da espécie.
\end{abstract}

Palavras-chave: ictiologia, mensuração, sistema digestório.

\section{DIGESTIVE TRACT ANATOMY OF THE SPECIES ACESTRORHYNCHUS LACUSTRIS (OSTARYOHISI, CHARACIFORMES) LÜTKEN, 1875 FROM ONDAS RIVER, WESTERN BAHIA, BRAZIL}

\begin{abstract}
This study aims to describe and measure the digestive tract of the species Acestrorhynchus lacustris. Ten specimens of the species were used, captured in different parts of the river basin of the Ondas river, located west of the State of Bahia, in the aquatic ecoregion of the São Francisco river. Fish measurements and weighing were carried out. The oropharyngeal cavity and postpharyngeal digestive organs were analyzed, described and measured: esophagus, stomach, pyloric cecum and intestine. To define the size of the organs of the species, regardless of the size of the sample, the relative length of the organs, except the pyloric cecum, was calculated. The species $A$. lacustris has a terminal mouth, a wide oral cleft and oropharyngeal cavity, conical and canine teeth; short, muscular, and compliant esophagus; elongated, muscular stomach, cecal type, shaped like a "Y"; seven to fifteen pyloric cecum

\footnotetext{
${ }^{1}$ Universidade Federal do Oeste da Bahia. *Correspondência: waltii@hotmail.com

${ }^{2}$ Universidade Federal de Viçosa. michelcm@ufv.br

${ }^{3}$ Universidade do Estado da Bahia. Imarinabpessoa@gmail.com
}

Guedes WF, Silva RM, Moreira MC, Pessoa LMB, Castro ERRS. Anatomia do tubo digestório da espécie Acestrorhynchus lacustris (Ostaryohisi, Characiformes) Lütken, 1875 do rio de Ondas, oeste da Bahia, Brasil. Vet. e Zootec. 2021; v28:001-015.
\end{abstract}


that appear as bowel movements; and intestine short, muscular and quite thick. The data obtained show that the size of the organs that make up the digestive tract of the species is not related to the size of the specimen. The study of the anatomical characteristics of the digestive tract expanded the biological and ecological knowledge of the studied species, helping to understand the performance of this species in natural ecosystems and in the elaboration of proposals for the preservation or management of the species.

Keywords: ichthyology, measurement, digestive system.

\section{ANATOMÍA DEL TUBO DIGESTORIAL DE LA ESPECIE ACESTRORHYNCHUS LACUSTRIS (OSTARYOHISI, CHARACIFORMES) LÜTKEN, 1875 DE RIO DE ONDAS, OESTE DE BAHIA, BRASIL}

\section{RESUMEN}

Este estudio tiene como objetivo describir y medir el tubo digestivo de la especie Acestrorhynchus lacustris. Se utilizaron diez ejemplares de la especie, capturado en diferentes partes de la cuenca hidrográfica del río del Ondas, al oeste del Estado de Bahía, en la ecorregión acuática del río São Francisco. Se realizaron mediciones y pesaje de peces. Se analizaron, describieron y midieron la cavidad bucofaríngea y los órganos digestivos posfaríngeos: esófago, estómago, ciego pilórico e intestino. Para definir el tamaño de los órganos de la especie, independientemente del tamaño de la muestra, se calculó la longitud relativa de los órganos, excepto el ciego pilórico. La especie A. lacustris tiene boca terminal, hendidura bucal amplia y cavidad bucofaríngea, dientes cónicos y caninos; esófago corto, musculoso y distensible; estómago alargado, musculoso, tipo cecal, en forma de "Y"; de siete a quince ciegos pilóricos que aparecen como evacuaciones intestinales; e intestino corto, musculoso y bastante grueso. Los datos obtenidos demuestran que el tamaño de los órganos que componen el tubo digestivo de la especie no está relacionado con el tamaño del espécimen. El estudio de las características anatómicas del tubo digestivo amplió el conocimiento biológico y ecológico de las especies estudiadas, ayudando a comprender el desempeño de esta especie en ecosistemas naturales y en la elaboración de propuestas para la preservación o manejo de la especie.

Palabras clave: ictiología, medición, aparato digestivo.

\section{INTRODUÇÃO}

O grupo dos peixes apresenta diversas variações nas estruturas básicas do sistema digestório, estando intrinsicamente relacionadas ao tipo de alimento consumido e ao ambiente, aspectos estes que influenciam na presença, posição e morfometria de um órgão em particular desse sistema (1).

O sistema digestório dos peixes é composto pelas estruturas que vão da boca ao ânus, sendo subdividido em cavidade bucofaríngea, denominada assim por formar uma única cavidade com a região bucal e a região faringeana, onde o alimento é selecionado, apreendido e conduzido até o esôfago, sendo este um órgão quase sempre curto, largo, reto e musculoso, com função de degustar o alimento e transportá-lo até o estômago. No estômago, o alimento é temporariamente armazenado, triturado e ocorre o início da digestão. Os outros órgãos são os cecos pilóricos, estrutura formada por várias vilosidades que aumenta a superfície intestinal, o intestino, onde ocorre a maior parte da absorção dos nutrientes e digestão, e o reto, onde é armazenado o bolo fecal (2). 
A espécie Acestrorhynchus lacustris pertence à família Characidae e a subfamília Acestrorhynchinae, que engloba apenas o gênero Acestrorhynchus, contendo 14 espécies (3). É conhecida popularmente como peixe-cachorro (4), possui hábito alimentar piscívoro $(5,6) \mathrm{e}$ está distribuída restritamente na América do Sul, ocorrendo principalmente nas bacias do Amazonas, Orinoco e nas drenagens do rio São Francisco, Paraná, Paraguai e La Plata (7).

$\mathrm{Na}$ bacia do rio de Ondas, localizada na ecorregião aquática do rio São Francisco, ainda são escassos os estudos que contemplem a ictiofauna local, de modo que o presente trabalho teve como objetivo descrever e mensurar o tubo digestório da espécie Acestrorhynchus lacustris, visando auxiliar na compreensão do desempenho desta espécie em ecossistemas naturais e na elaboração de propostas para a preservação ou manejo da espécie.

\section{MATERIAIS E MÉTODOS}

\section{Coleta de exemplares}

Foram utilizados 10 exemplares da espécie Acestrorhynchus lacustris, capturados no médio e alto curso do rio de Ondas e no médio curso do rio de Pedras. Estes rios fazem parte da bacia do rio de Ondas, que está localizada no extremo oeste do Estado da Bahia, entre as coordenadas de $11^{\circ} 55^{\prime}$ e $12^{\circ} 34^{\prime}$ de latitude sul e $46^{\circ} 23^{\prime}$ e $45^{\circ} 0^{\prime}$ de longitude oeste (8) (Figura 1), e faz parte da ecorregião aquática do rio São Francisco.

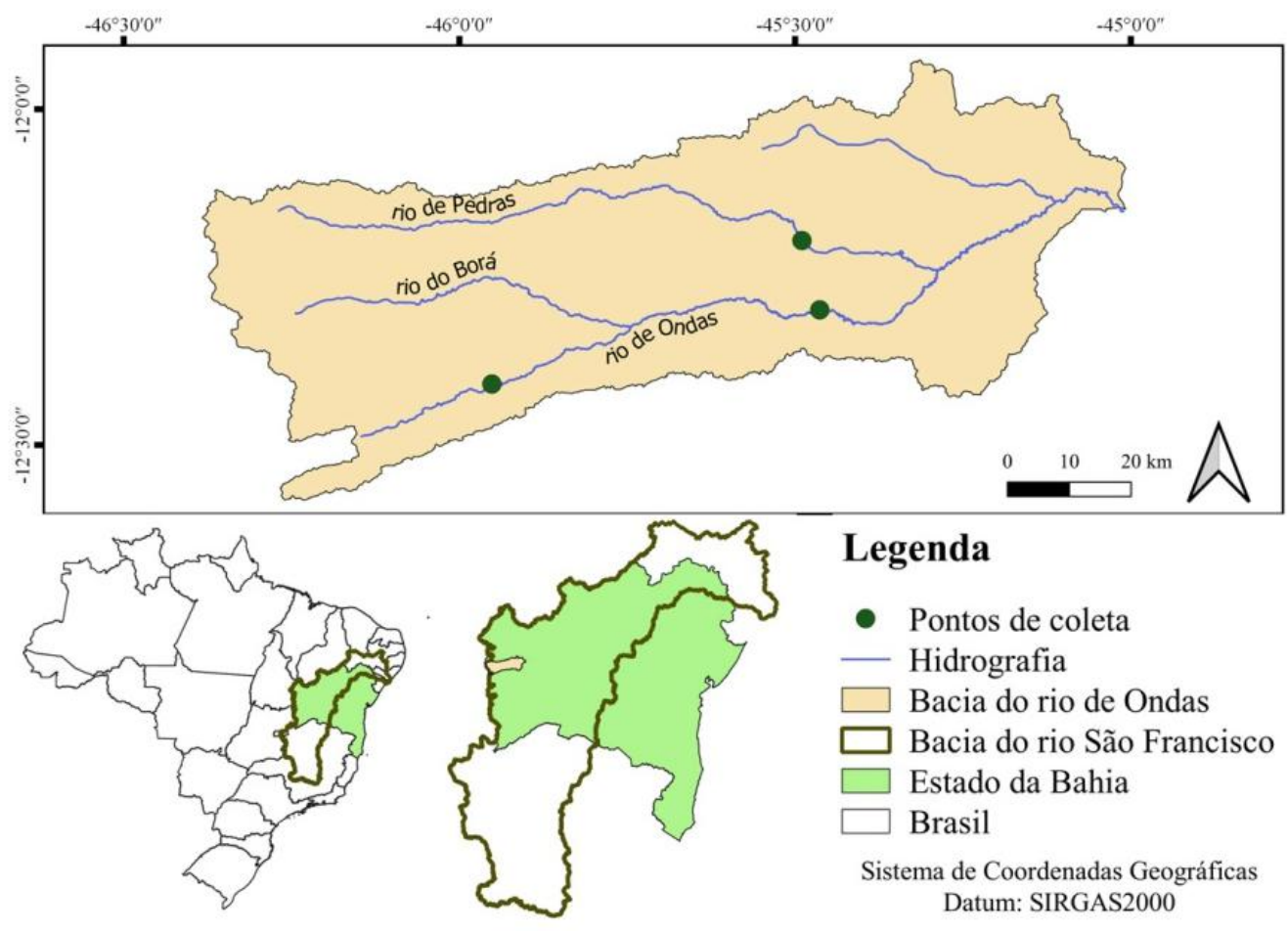

Figura 1. Bacia hidrográfica do rio de Ondas, oeste da Bahia, apresentando seus limites, municípios e trechos onde ocorreram as coletas da ictiofauna.

A escolha do método de coleta foi realizada de acordo com as características do local, como profundidade e largura do canal, além da velocidade da água. Em geral, utilizaram-se redes das malhas três, quatro, cinco, seis, sete, oito, nove, 10,11, 12 e 14 entre nós adjacentes, armadas por um período de 12 horas e verificação a cada 6 horas. 
Como sugerido por Uieda e Castro (9), os peixes capturados foram anestesiados com 3 $\mathrm{ml}$ da solução de eugenol $(100 \mathrm{mg} / \mathrm{ml})$, posteriormente, fixados em formaldeído $10 \%$, sendo etiquetados e acondicionados em sacos plásticos por um período máximo de 72 horas e, em seguida , transferidos para conservação em álcool $70 \%$.

Obteve-se autorização para coleta e transporte da ictiofauna no Sistema de Autorização e Informação em Biodiversidade (SISBIO), do Instituto Brasileiro do Meio Ambiente e dos Recursos Renováveis (IBAMA), sob a permissão para atividade com finalidade científica de número 66270-1, bem como a aprovação no Conselho de Ética Em Uso de Animal da Universidade Federal do Oeste da Bahia (UFOB), de número 0011/18.

\section{Análises morfológicas, mensuração dos peixes e seus tubos digestórios}

Após a coleta, os peixes foram armazenados em solução de álcool a 70\%, identificados e transportados para o Laboratório do Museu de Ciências do Cerrado Nordestino da Universidade Federal do Oeste da Bahia para realização da análise e mensuração de dados.

Para cada exemplar foi identificado o seu estágio de desenvolvimento, segundo a classificação de Vazzoler (10), sendo utilizados no estudo apenas animais adultos. Em todos os espécimes foram mensurados o comprimento padrão (extremidade cranial até o final do pedúnculo caudal), altura do corpo (obtida no início da nadadeira dorsal) e o peso corpóreo.

Os exemplares analisados foram numerados na sequência decrescente do seu comprimento padrão, sendo assim, o número um foi o que apresentou o maior comprimento padrão e o número 10 o menor. Todas as medidas de comprimento e altura foram tomadas com auxílio de paquímetro digital (Kingtools) e o peso utilizando-se balança digital.

Posteriormente uma incisão no sentido craniocaudal foi feita na linha mediana - ventral de cada exemplar, desde a boca até o ânus, contornando-o, para análise do tubo digestório.

$\mathrm{Na}$ cavidade bucofaríngea foram realizadas três mensurações: o comprimento da abertura bucal, em seu plano sagital mediano, conhecida como fenda bucal, verificando a distância entre os lábios ao abrir a boca sem distender a musculatura; o comprimento lateral da boca, analisando a distância entre o plano sagital mediano da boca e sua parte lateral; e o comprimento longitudinal do teto da cavidade, mensurando da boca ao início do esôfago. Foram analisadas, também, a forma e posição da boca e tipos de dentes, sendo o único anexo analisado no presente estudo, utilizando um microscópio estereoscópio e lupa.

$\mathrm{Na}$ sequência, os órgãos pós-faríngeos do tubo digestório, constituído pelo esôfago, estômago, cecos pilóricos, intestino e reto, foram retirados, descritos e mensurados. O reto não foi analisado separadamente do intestino, pois não foi possível diferenciar macroscopicamente o reto do intestino, nos tubos digestórios avaliados.

No estômago, foram realizadas três mensurações: o comprimento longitudinal (região mais alongada, que se estende da região cárdia até a fúndica); a região pilórica (do início da curvatura até o piloro); e o comprimento transversal do órgão (tendo como ponto referencial a curvatura da região pilórica), como identificado, respectivamente pelas letras $\mathrm{A}, \mathrm{B}$ e $\mathrm{C}$, na Figura 2.

O comprimento dos cecos pilóricos foi obtido levando em consideração a distância da base até o ápice do saco cego. Os cecos com ramificações tiveram o comprimento do pedúnculo comum às ramificações inclusos na mensuração do seu comprimento, visando uniformizar as medidas, seguindo metodologia realizada por Seixas Filho et al. (11). Além da mensuração do comprimento, os cecos foram contabilizados.

A descrição e a disposição das estruturas anatômicas foram realizadas conforme os planos anatômicos de delimitação, seguindo a nomenclatura anatômica proposta por Constantinescu et al. (12).

Guedes WF, Silva RM, Moreira MC, Pessoa LMB, Castro ERRS. Anatomia do tubo digestório da espécie Acestrorhynchus lacustris (Ostaryohisi, Characiformes) Lütken, 1875 do rio de Ondas, oeste da Bahia, Brasil. Vet. e Zootec. 2021; v28:001-015. 


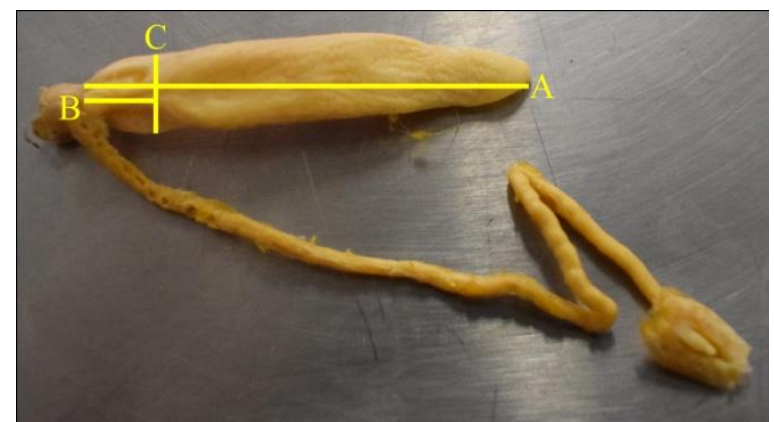

Figura 2. Estômago de um dos exemplares da espécie A. lacustris, demonstrando os três pontos de mensurações: A) comprimento longitudinal; B) região pilórica; e C) comprimento transversal do órgão.

\section{Análise dos dados}

Para as análises dos dados obtidos foi utilizada a média e o desvio padrão das mensurações realizadas nos espécimes, conforme as equações (13):

$$
\begin{aligned}
& \overline{\mathrm{x}}=\frac{\sum_{\mathrm{j}=0}^{\mathrm{n}} \mathrm{x}_{\mathrm{j}}}{\mathrm{n}} \overline{\mathrm{x}}=\frac{\sum_{\mathrm{j}=0}^{\mathrm{n}} \mathrm{x}_{\mathrm{j}}}{\mathrm{n}} \overline{\mathrm{x}}=\frac{\sum_{\mathrm{i}=0}^{\mathrm{n}} \mathrm{x}_{\mathrm{i}}}{\mathrm{n}} \\
& \mathrm{s}=\sqrt{\frac{\sum_{i=0}^{\mathrm{n}}\left(x_{i}-\bar{x}\right)^{2}}{\mathrm{n}-1}} \mathrm{~s}=\sqrt{\frac{\sum_{i=0}^{\mathrm{n}}\left(x_{i}-\bar{x}\right)^{2}}{\mathrm{n}-1}}
\end{aligned}
$$

em que:

$$
\begin{array}{lll}
\overline{\mathrm{xx}} \overline{\mathrm{x}} & =\text { média do conjunto de valores amostrais; } \\
\mathrm{X}_{\mathrm{i}} & =\text { valor amostral; } \\
\mathrm{n} & =\text { número de valores amostrais; } \mathrm{e} \\
\mathrm{S} & =\text { desvio padrão. }
\end{array}
$$

Para se obter a média e o desvio padrão dos órgãos da espécie independente do tamanho de cada exemplar, foram calculados os comprimentos relativos, dados pela divisão do comprimento do órgão em análise pelo comprimento padrão do exemplar $(14,15)$.

As análises relativas foram feitas para a cavidade bucofaríngea, utilizando as três medidas, esôfago, estômago, também utilizando as três mensurações, e o intestino de cada exemplar.

\section{RESULTADOS E DISCUSSÕES}

Os 10 exemplares analisados apresentaram gônadas ocupando mais de 2/3 da cavidade celomática (Tabela 1), abrangendo grande parte da cavidade, sendo classificados no estágio maduro (Figura 3), conforme os critérios de Vazzoler (10). Dessa forma, todos os indivíduos utilizados neste estudo apresentaram mesmo estágio de desenvolvimento, sendo considerados adultos.

Segundo Seixas Filho (11), a anatomia do tubo digestório dos peixes está relacionada ao estágio de desenvolvimento do indivíduo, pelas adaptações e modificações dos órgãos e estruturas que o compõe. Assim, foram selecionados indivíduos no mesmo estágio a fim de permitir uma melhor descrição anatômica. 
Tabela 1. Comprimento da cavidade celomática e gônadas dos exemplares da espécie $A$. lacustris.

\begin{tabular}{cccc}
\hline Exemplar & $\begin{array}{c}\text { Cavidade Celomática } \\
(\mathbf{m m})\end{array}$ & $\begin{array}{c}\text { Comprimento da } \\
\text { gônada direita } \\
(\mathbf{m m})\end{array}$ & $\begin{array}{c}\text { Comprimento da } \\
\text { gônada esquerda } \\
(\mathbf{m m})\end{array}$ \\
\hline 1 & 96,1 & 69,53 & 70,21 \\
\hline 2 & 93,85 & 69,2 & 68,01 \\
\hline 3 & 89,52 & 67,79 & 67,91 \\
\hline 4 & 89,03 & 73,46 & 71,14 \\
\hline 5 & 97,08 & 68,03 & 65,12 \\
\hline 6 & 82,13 & 55,48 & 59,53 \\
\hline 7 & 79,4 & 65,07 & 58,63 \\
\hline 8 & 80,73 & 58,58 & 57,25 \\
\hline 9 & 79,04 & 55,96 & 52,6 \\
\hline 10 & 71,85 & 57,99 & 56,87 \\
\hline
\end{tabular}

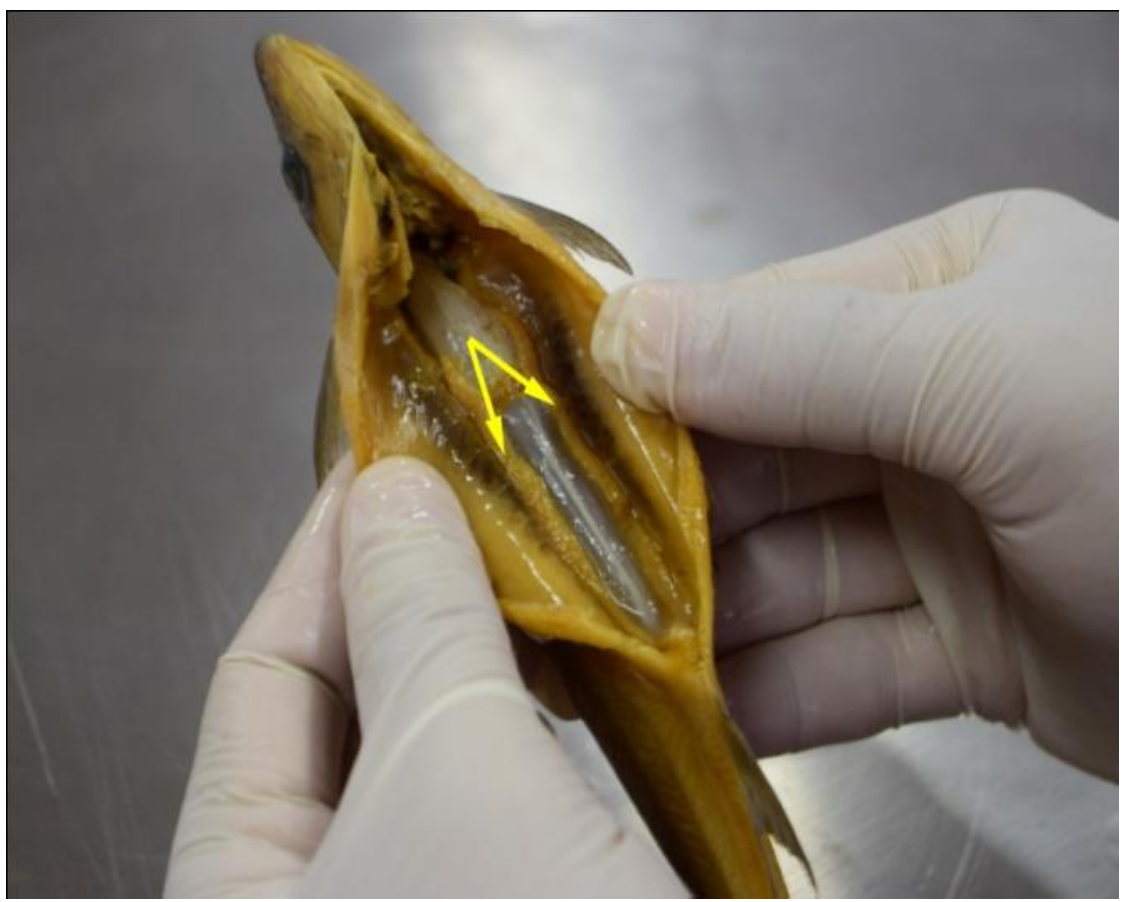

Figura 3. Cavidade celomática de um dos exemplares de A. lacustris, apresentando as gônadas ocupando grande parte da cavidade.

Nos espécimes estudados a boca terminal estende-se até os lados da cabeça, com fenda bucal ampla e horizontalmente desenvolvida, focinho longo, e pré-maxilar não protrátil, o que permite uma maior firmeza ao capturar o alimento. De acordo com Rotta (2), uma ampla fenda bucal e boca estendendo até os lados da cabeça são características de peixes que se alimentam, preferencialmente, de organismos animais, permitindo capturar e ingerir grandes presas inteiras. O que pôde ser confirmado no presente estudo, uma vez que no estômago de um dos exemplares foi encontrado um peixe inteiro.

A cavidade bucofaríngea dos espécimes apresentou-se ampla no plano caudal do órgão, afunilando rostralmente, com dentição desenvolvida, apresentando apenas dentes cônicos e caninos (Figura 4). 


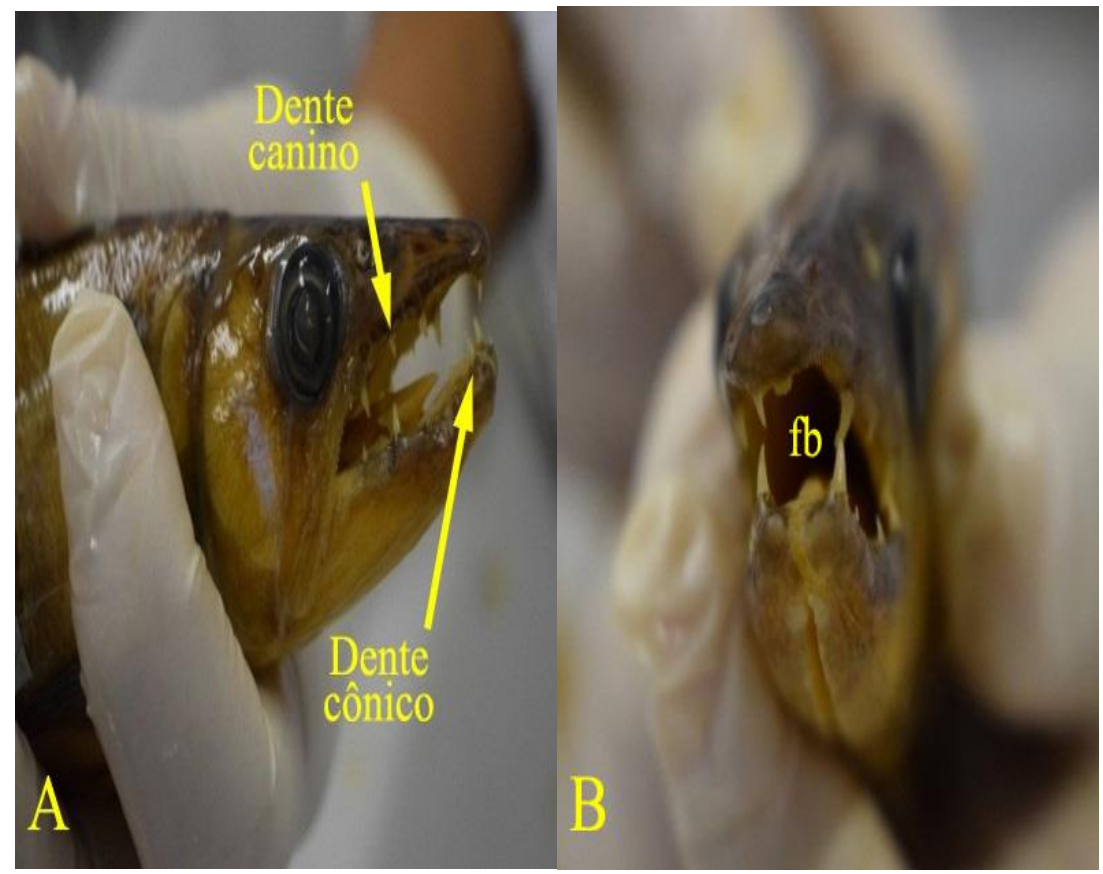

Figura 4. Boca de um dos exemplares de A. lacustris: A) vista lateral esquerda, evidenciando os dentes cônicos e caninos; e B) vista frontal, apresentando a fenda bucal (fb).

Segundo Britski (16), a cavidade bucofaríngea da espécie é caracterizada pela presença de pequenos dentes no palato, duas séries, sendo uma característica que diferencia a espécie de outros carnívoros, conforme foi evidenciada nos exemplares analisados (Figura 5).

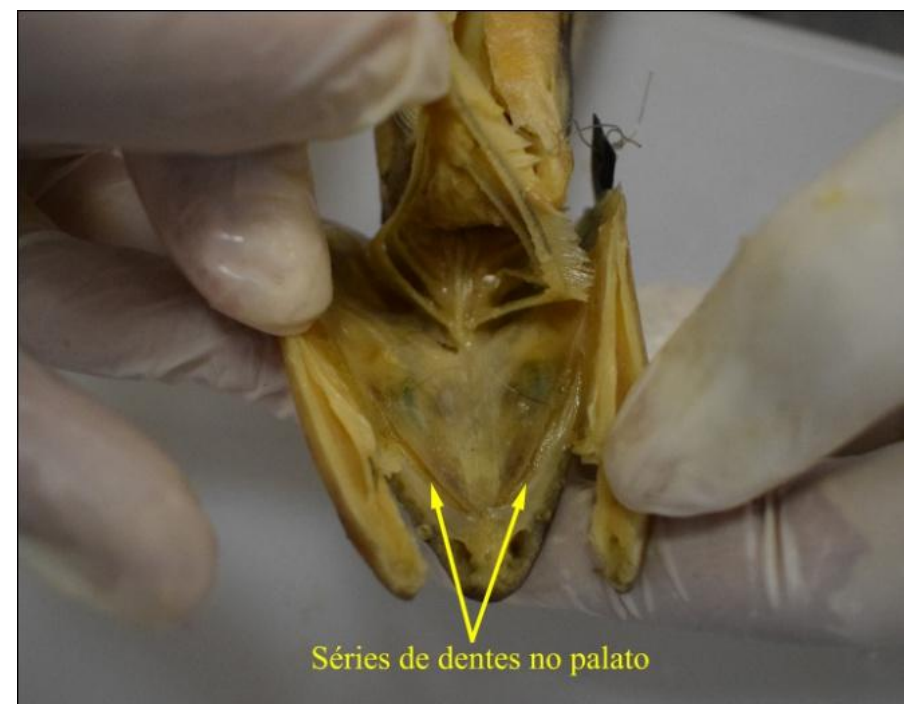

Figura 5. Teto da cavidade bucofaríngea de um dos exemplares de A. lacustris, apresentando duas séries de dentes no palato (setas).

O esôfago dos exemplares é curto, bastante musculoso, características descritas por Benedito (17) e Rotta (2) para o grupo dos peixes, e com musculatura aparentemente de fácil distensão (Figura 6), tendo sido o limite entre o esôfago e o estômago verificado macroscopicamente com a mudança da espessura dos órgãos. 


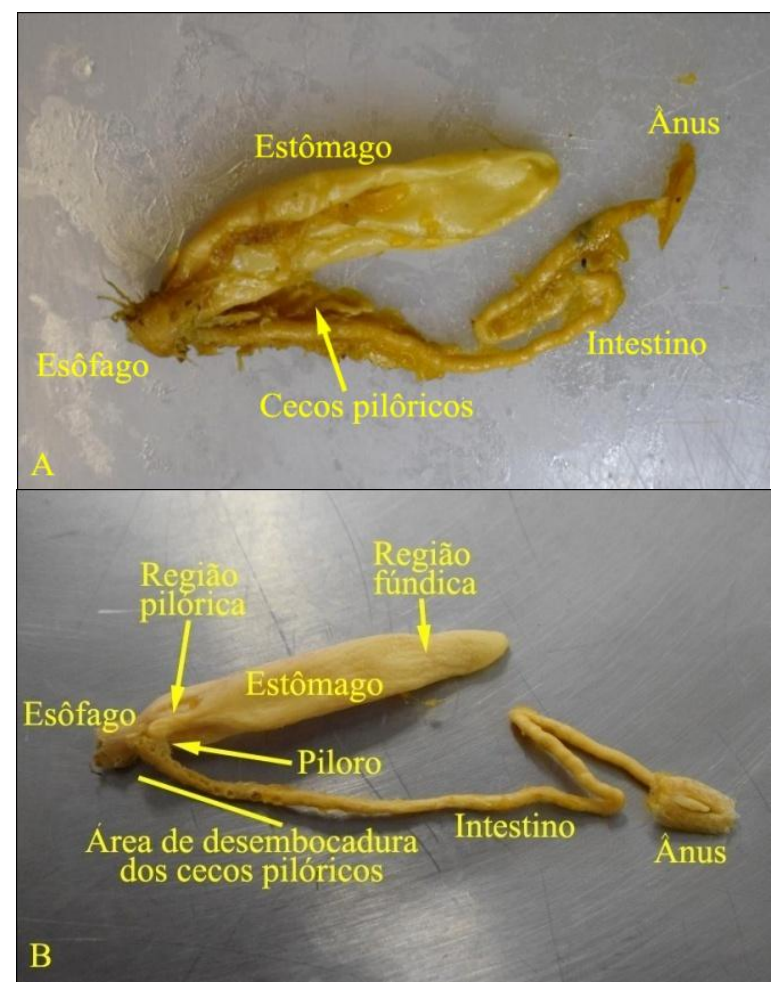

Figura 6. Órgãos pós-faríngeos do tubo digestório de um dos exemplares de A. lacustris: A) disposição dos órgãos na cavidade celomática; e B) anatomia dos órgãos após a retirada do excesso de gordura e dos cecos pilóricos.

Nos exemplares analisados foi observado estômago alongado, bastante musculoso, elástico, do tipo cecal, com formato de "Y", apresentando uma região pilórica curta, tubular e se formando paralelamente à região cárdica, próxima ao esôfago, enquanto a região fúndica apresentou-se ampla e terminando em fundo cego (Figura 6). A região fúndica é alongada, proporcionando a ingestão de presas inteiras (18), como evidenciado no estômago de um dos exemplares analisados da espécie (Figura 7).

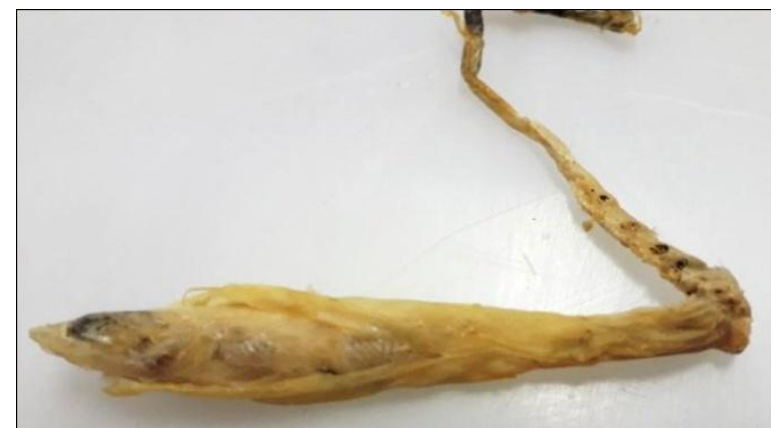

Figura 7. Estômago de um dos exemplares de A. lacustris evidenciando a ingestão de um peixe.

Foram verificados poucos cecos pilóricos nos espécimes analisados, variando de sete a 15, curtos, dispostos sobre o estômago, evaginados apenas no intestino e localizados distalmente ao piloro, como na maioria dos teleósteos, conforme descrito por Liem (19). Estes cecos pilóricos abrangem uma grande área do intestino, por estarem dispostos em uma única fileira (Figuras 6).

Identificou-se nos exemplares a existência de intestino curto, musculoso, bastante espesso, não enovelado e com algumas dobras na parte final do órgão. Como é um órgão 
curto, apresenta disposição contínua na cavidade celomática, com uma pequena aglomeração no final da cavidade, devido às dobras (Figura 6). Segundo Rotta (2), os peixes carnívoros possuem, basicamente, intestino curto, reto e espesso, com maior número de dobras, que aumentam a eficiência de absorção dos nutrientes ingeridos, características observadas na espécie.

De acordo com Benedito (17), o tamanho e forma da boca, a existência ou não de dentes, o comprimento, formato e tamanho do estômago e intestino podem variar conforme o hábito alimentar da espécie.

Os dados de biometria mensurados para os espécimes estudados estão dispostos na Tabela 2.

Tabela 2. Comprimento padrão, altura e peso dos exemplares analisados da espécie $A$. lacustris e suas respectivas médias e desvio padrão.

\begin{tabular}{cccc}
\hline Exemplar & $\begin{array}{c}\text { Comprimento padrão } \\
(\mathbf{m m})\end{array}$ & $\begin{array}{c}\text { Altura } \\
(\mathbf{m m})\end{array}$ & $\begin{array}{c}\text { Peso } \\
(\mathbf{g})\end{array}$ \\
\hline 1 & 232,19 & 53,46 & 158 \\
\hline 2 & 210,12 & 48,86 & 146 \\
\hline 3 & 206,54 & 46,19 & 117 \\
\hline 4 & 204,75 & 44,56 & 111 \\
\hline 5 & 198,4 & 42,33 & 98 \\
\hline 6 & 183,11 & 39,21 & 71 \\
\hline 7 & 177,42 & 38,98 & 65 \\
\hline 8 & 177,05 & 41,82 & 73 \\
\hline 9 & 169,84 & 36,06 & 61 \\
\hline 10 & 165,61 & 38 & 58 \\
\hline Média & 192,50 & 42,95 & 95,80 \\
\hline Desvio padrão & 21,24 & 5,39 & 36,21 \\
\hline
\end{tabular}

Nos exemplares analisados não foram retirados o bolo alimentar, pois poderia romper a parede dos órgãos, sendo analisados de acordo com as condições naturais em que foram coletados, podendo explicar as divergências no peso e comprimento dos exemplares e de alguns órgãos.

A cavidade bucofaríngea é longa e com grande fenda bucal, com média e desvio padrão da abertura bucal de 13,51 $\pm 1,55 \mathrm{~mm}$, sendo uma medida que não seguiu uma proporção com o comprimento padrão.

Em relação ao comprimento lateral da boca, apresentaram uma média de 23,66 mm com desvio padrão de 4,51 mm. Essa medida não apresentou relação com o tamanho do peixe, pois os exemplares de números um, cinco, sete e nove apresentaram comprimento lateral da boca maior que os exemplares anteriores, com maior comprimento padrão.

Quanto ao comprimento longitudinal do órgão, os exemplares apresentaram em média $46,88 \pm 6,53 \mathrm{~mm}$. Observaram-se que as medidas desse parâmetro também não demonstraram relação com a sequência do comprimento padrão dos exemplares, uma vez que, os exemplares seis e sete possuem comprimento longitudinal maior que o exemplar cinco, que possui maior comprimento padrão, assim como o exemplar $10 \mathrm{em}$ relação ao nove (Tabela 3 ). Os resultados revelam que a abertura bucal e o comprimento longitudinal não estão proporcionais ao comprimento padrão da espécie, mas são bem amplos, permitindo o consumo de grandes presas.

Guedes WF, Silva RM, Moreira MC, Pessoa LMB, Castro ERRS. Anatomia do tubo digestório da espécie Acestrorhynchus lacustris (Ostaryohisi, Characiformes) Lütken, 1875 do rio de Ondas, oeste da Bahia, Brasil. Vet. e Zootec. 2021; v28:001-015. 
Os esôfagos dos exemplares analisados apresentaram média de 5,79 $\mathrm{mm} \pm 1,32 \mathrm{~mm}$. O comprimento do órgão não demonstrou correlação com o tamanho do peixe, uma vez que exemplares menores apresentaram esôfago maior, como ocorreu nos exemplares dois, três, seis e nove.

Tabela 3. Medidas da cavidade bucofaríngea (CBF) dos exemplares analisados da espécie $A$. lacustris e suas respectivas médias e desvio padrão.

\begin{tabular}{cccc}
\hline Exemplar & $\begin{array}{c}\text { Fenda bucal } \\
(\mathbf{m m})\end{array}$ & $\begin{array}{c}\text { Comprimento lateral da } \\
\text { boca }(\mathbf{m m})\end{array}$ & $\begin{array}{c}\text { Comprimento longitudinal da } \\
\text { CBF (mm) }\end{array}$ \\
\hline 1 & 13,78 & 30,95 & 57,26 \\
\hline 2 & 12,53 & 28,53 & 53,96 \\
\hline 3 & 12,85 & 28,98 & 52,6 \\
\hline 4 & 12,57 & 23,75 & 50,28 \\
\hline 5 & 16,52 & 24,98 & 43,6 \\
\hline 6 & 12,76 & 19,57 & 46,18 \\
\hline 7 & 15,29 & 21,18 & 46,38 \\
\hline 8 & 14,82 & 19,29 & 41,35 \\
\hline 9 & 11,69 & 20,78 & 37,45 \\
\hline 10 & 12,29 & 18,59 & 39,75 \\
\hline Média & 13,51 & 23,66 & 46,88 \\
\hline Desvio padrão & 1,55 & 4,51 & 6,53 \\
\hline
\end{tabular}

Os estômagos dos 10 exemplares estudados tiveram três mensurações: comprimento longitudinal, região pilórica e comprimento transversal. A média do comprimento longitudinal do estômago nos espécimes foi de 53,91 $\pm 12,24 \mathrm{~mm}$, demonstrando que variou bastante entre os exemplares, a região pilórica teve média de 7,28 $\pm 1,69 \mathrm{~mm}$, e o comprimento transversal, de $11,93 \pm 2,83 \mathrm{~mm}$. Os altos valores de desvio padrão no comprimento longitudinal e transversal podem ser explicados devido ao estado de repleção do órgão de alguns exemplares, pois alguns foram analisados com o estômago quase vazio, enquanto outros o órgão estava cheio. As medidas realizadas no estômago não apresentaram correlação com o comprimento padrão dos exemplares.

A espécie A. lacustris apresentou em média 12 cecos pilóricos, variando de sete a 15 entre os exemplares, com comprimento médio de 7,54 $\pm 1,29 \mathrm{~mm}$. Essa variação confirma a descrição realizada por Baldisserotto (20), ao afirmar que a quantidade e forma dos cecos pilóricos podem variar de espécie para espécie e entre exemplares da mesma espécie.

Os cecos pilóricos são de diversos tamanhos, não apresentando uma ordem crescente ou decrescente em cada exemplar ou entre os exemplares, com grandes variações. Os exemplares não demonstraram relação entre a quantidade de cecos e o tamanho do animal, mostrando que peixes pequenos podem apresentar quantidade de cecos parecida com peixes maiores e que a espécie não apresenta uma quantidade padrão de cecos pilóricos (Tabela 4).

Os cecos pilóricos da espécie estão dispostos no intestino, ocupando em média $19,51 \mathrm{~mm}$ do órgão, correspondendo a cerca de $21 \%$ do órgão. A área do intestino com cecos também não apresentou correlação com o comprimento padrão dos exemplares, além de não demonstrar relação com a quantidade de cecos pilóricos e com o tamanho do intestino, pois o exemplar três apresentou a maior área do intestino com cecos, mas não apresentou o maior intestino e número de cecos. Essa divergência ocorre porque os cecos apresentam diferentes tamanhos e distâncias entre si.

O intestino dos exemplares apresentou média no comprimento de $91,46 \mathrm{~mm} \pm$ $31,79 \mathrm{~mm}$, com grande variação. Uma correlação entre o comprimento do intestino e o comprimento padrão não foi verificada. Os exemplares dois, quatro, cinco, seis, nove e 10 tiveram o intestino maior do que exemplares maiores. $\mathrm{O}$ exemplar 10 apresentou o terceiro 
maior intestino entre os exemplares, permitindo inferir que indivíduos pequenos podem ter grandes intestinos, ou seja, o tamanho do órgão não está relacionado com o tamanho do peixe.

Tabela 4. Comprimento e quantidade dos cecos pilóricos (CP) dos exemplares analisados da espécie A. lacustris.

\begin{tabular}{|c|c|c|c|c|c|c|c|c|c|c|c|c|c|c|c|}
\hline \multirow{2}{*}{ Exemplar } & CP1 & CP2 & CP3 & CP4 & CP5 & CP6 & CP7 & CP8 & CP9 & CP10 & CP11 & CP12 & CP13 & CP14 & CP15 \\
\hline & \multicolumn{15}{|c|}{ Milímetros } \\
\hline 1 & 9,9 & 10,01 & 6,64 & 8,08 & 9,06 & 9,16 & 8,19 & 8,7 & 7,54 & 8,74 & 7,98 & 8,71 & 11,3 & & \\
\hline 2 & 6,01 & 7,23 & 7,67 & 9,21 & 11,56 & 5,75 & 12,03 & & & & & & & & \\
\hline 3 & 5,09 & 6,59 & 7,41 & 9,85 & 8,66 & 9,2 & 6,58 & 7,85 & 9,3 & 8,98 & 7,64 & 8,97 & 6,56 & & \\
\hline 4 & 8,25 & 5,39 & 5,98 & 6,02 & 6,58 & 6,19 & 6,05 & 6,77 & 5,75 & 6,44 & 6,5 & 5,36 & 6,09 & 8,72 & \\
\hline 5 & 5,86 & 10,02 & 9,8 & 7,64 & 8,55 & 9,81 & 10,52 & 10,22 & 10,42 & 8,27 & 8,33 & 10,18 & 8,27 & 8,42 & 7,91 \\
\hline 6 & 10,12 & 7,77 & 9,2 & 9,92 & 10,39 & 9,5 & 8,68 & 8,82 & 8,58 & 7,82 & 8,9 & 8,71 & & & \\
\hline 7 & 5,41 & 7,04 & 7,68 & 7,53 & 8,87 & 7,84 & 7,88 & 8,58 & 7,05 & & & & & & \\
\hline 8 & 7,38 & 5,37 & 5,91 & 5,38 & 6,63 & 7,61 & 7,31 & 7,47 & 5,81 & 5,25 & & & & & \\
\hline 9 & 6,16 & 4,47 & 5,62 & 5,43 & 4,77 & 4,95 & 5,34 & 5,7 & 4,97 & 5,68 & 5,91 & 7,23 & 4,68 & 4,91 & \\
\hline 10 & 6,31 & 6,04 & 6,17 & 7,04 & 6,98 & 5,28 & 6,75 & 6,8 & 5,48 & 6,97 & 6,95 & 5,33 & 7,76 & & \\
\hline
\end{tabular}

Os dados referentes ao comprimento dos órgãos digestórios pós-faringeos dos exemplares estudados estão apresentados na Tabela 5.

Tabela 5. Comprimento e quantidade dos órgãos digestórios pós-faringeos dos exemplares analisados da espécie A. lacustris.

\begin{tabular}{|c|c|c|c|c|c|c|c|c|}
\hline \multirow[t]{2}{*}{ Exemplar } & Esôfago & $\begin{array}{c}\text { Comprimento } \\
\text { longitudinal do } \\
\text { estômago }\end{array}$ & $\begin{array}{c}\text { Estômago - } \\
\text { região } \\
\text { pilórica }\end{array}$ & $\begin{array}{c}\text { Comprimento } \\
\text { transversal do } \\
\text { estômago }\end{array}$ & $\begin{array}{l}\text { Quantidade } \\
\text { de cecos } \\
\text { pilóricos }\end{array}$ & $\begin{array}{c}\text { Média do } \\
\text { comprimento } \\
\text { dos cecos } \\
\text { pilóricos } \\
\end{array}$ & Intestino & $\begin{array}{c}\text { Área do } \\
\text { intestino } \\
\text { com cecos } \\
\text { pilóricos } \\
\end{array}$ \\
\hline & \multicolumn{8}{|c|}{ Milímetros } \\
\hline 1 & 6,9 & 80,92 & 9,77 & 16,07 & 13 & 8,77 & 89,87 & 17,42 \\
\hline 2 & 7,88 & 56,61 & 10,09 & 14,21 & 7 & 8,49 & 148,91 & 23,24 \\
\hline 3 & 7,24 & 49,36 & 7,61 & 13,6 & 13 & 7,9 & 74,5 & 26,16 \\
\hline 4 & 6,9 & 56,55 & 8,83 & 13,48 & 14 & 6,44 & 83,89 & 16,84 \\
\hline 5 & 5,15 & 58,68 & 6,33 & 12,93 & 15 & 8,95 & 123,83 & 22,29 \\
\hline 6 & 5,16 & 46,35 & 6,38 & 8,67 & 12 & 9,03 & 102,05 & 20,78 \\
\hline 7 & 5,01 & 52,41 & 5,96 & 6,94 & 9 & 7,54 & 51,39 & 12,56 \\
\hline 8 & 4,54 & 47,09 & 6,19 & 10,42 & 10 & 6,41 & 44,95 & 11,99 \\
\hline 9 & 5,14 & 33,02 & 6,04 & 9,87 & 14 & 5,42 & 82,73 & 20,89 \\
\hline 10 & 3,98 & 58,15 & 5,55 & 13,09 & 13 & 6,45 & 112,5 & 22,95 \\
\hline Média & 5,79 & 53,91 & 7,28 & 11,93 & 12 & 7,54 & 91,46 & 19,51 \\
\hline $\begin{array}{l}\text { Desvio } \\
\text { padrão }\end{array}$ & 1,32 & 12,24 & 1,69 & 2,83 & 2,54 & 1,29 & 31,79 & 4,69 \\
\hline
\end{tabular}

Segundo Baldisserotto (20), peixes carnívoros não possuem a capacidade de alterar a estrutura e as propriedades absortivas do seu sistema digestório, pois a dieta não varia de forma significativa, ocorrendo apenas em peixes herbívoros ou onívoros. Este argumento, no entanto, não explica a falta de correlação entre o tamanho dos órgãos e o comprimento padrão dos exemplares da espécie, podendo-se constatar, apenas, a influência do bolo alimentar, presente ou ausente, na mensuração de alguns órgãos.

Para verificar a medida de cada órgão para a espécie, independentemente do tamanho dos exemplares, foi verificado o comprimento relativo (Tabela 6). Em relação as medidas realizadas na cavidade bucofaríngea, a fenda bucal apresentou comprimento relativo com média de $0,07 \mathrm{~mm} \pm 0,01 \mathrm{~mm}$, a área lateral teve $0,12 \mathrm{~mm} \pm 0,01 \mathrm{~mm}$, e o teto da cavidade bucofaríngea com $0,24 \mathrm{~mm} \pm 0,01 \mathrm{~mm}$, apontando um baixo desvio padrão. 
O esôfago apresentou comprimento relativo de $0,03 \mathrm{~mm} \pm 0,004 \mathrm{~mm}$, com baixa variação entre os exemplares. $\mathrm{O}$ estômago, que teve três mensurações, comprimento longitudinal, região pilórica e comprimento transversal, apresentou $0,28 \mathrm{~mm} \pm 0,05 \mathrm{~mm}, 0,04$ $\mathrm{mm} \pm 0,005 \mathrm{~mm}$ e $0,06 \mathrm{~mm} \pm 0,01 \mathrm{~mm}$, como respectivas médias de comprimento relativo. Com relação ao intestino, o órgão apresentou a média do comprimento relativo de $0,48 \mathrm{~mm} \pm$ $0,15 \mathrm{~mm}$, sendo o órgão com maior variação.

Tabela 6. Comprimento Relativo (CR) da cavidade bucofaríngea (CBF), esôfago, estômago e intestino analisados da espécie A. lacustris.

\begin{tabular}{|c|c|c|c|c|c|c|c|c|}
\hline \multirow[t]{2}{*}{ Exemplar } & $\begin{array}{c}\text { CR da fenda } \\
\text { bucal }\end{array}$ & $\begin{array}{c}\text { CR da } \\
\text { área } \\
\text { lateral da } \\
\text { boca } \\
\end{array}$ & $\begin{array}{c}\text { CR } \\
\text { da } \\
\text { CBF }\end{array}$ & $\begin{array}{c}\text { CR do } \\
\text { esôfago }\end{array}$ & $\begin{array}{l}\text { CR do comprimento } \\
\text { longitudinal do } \\
\text { estômago }\end{array}$ & $\begin{array}{l}\text { CR da região } \\
\text { pilórica do } \\
\text { estômago }\end{array}$ & $\begin{array}{c}\text { CR do } \\
\text { comprimento } \\
\text { transversal do } \\
\text { estômago } \\
\end{array}$ & $\begin{array}{c}\text { CR do } \\
\text { intestino }\end{array}$ \\
\hline & \multicolumn{8}{|c|}{ Milímetros } \\
\hline 1 & 0,06 & 0,13 & 0,25 & 0,03 & 0,35 & 0,04 & 0,07 & 0,39 \\
\hline 2 & 0,06 & 0,14 & 0,26 & 0,04 & 0,27 & 0,05 & 0,07 & 0,71 \\
\hline 3 & 0,06 & 0,14 & 0,25 & 0,04 & 0,24 & 0,04 & 0,07 & 0,36 \\
\hline 4 & 0,06 & 0,12 & 0,25 & 0,03 & 0,28 & 0,04 & 0,07 & 0,41 \\
\hline 5 & 0,08 & 0,13 & 0,22 & 0,03 & 0,3 & 0,03 & 0,07 & 0,62 \\
\hline 6 & 0,07 & 0,11 & 0,25 & 0,03 & 0,25 & 0,03 & 0,05 & 0,56 \\
\hline 7 & 0,09 & 0,12 & 0,26 & 0,03 & 0,3 & 0,03 & 0,04 & 0,29 \\
\hline 8 & 0,08 & 0,11 & 0,23 & 0,03 & 0,27 & 0,03 & 0,06 & 0,25 \\
\hline 9 & 0,07 & 0,12 & 0,22 & 0,03 & 0,19 & 0,04 & 0,06 & 0,49 \\
\hline 10 & 0,07 & 0,11 & 0,24 & 0,02 & 0,35 & 0,03 & 0,08 & 0,68 \\
\hline Média & 0,07 & 0,12 & 0,24 & 0,03 & 0,28 & 0,04 & 0,06 & 0,48 \\
\hline $\begin{array}{l}\text { Desvio } \\
\text { padrão }\end{array}$ & 0,01 & 0,01 & 0,01 & 0,004 & 0,05 & 0,005 & 0,01 & 0,15 \\
\hline
\end{tabular}

O comprimento relativo do intestino da espécie A. lacustris diverge do encontrado por Amaral (21), ao analisar a anatomia de duas espécies do gênero Acestrorhynchus, coletadas no rio São Francisco, verificando que a espécie A. lacustris apresenta comprimento relativo do intestino de 0,66 , enquanto os exemplares analisados apresentaram em média comprimento relativo de 0,48 , constatando uma pequena diferença entre os resultados obtidos. Essa divergência sugere que o ambiente influencia na morfologia do órgão ou que a espécie sofreu modificações ao longo do tempo.

Os peixes carnívoros apresentam comprimento relativo do intestino variando entre 0,2 a 2,5, os onívoros entre 0,6 e 8,0 e os herbívoros de 0,8 a 15,0 (2). Seguindo esses valores, a média do comprimento relativo do intestino da espécie estudada permite inferir que possui hábito alimentar carnívoro. Segundo o mesmo autor, uma fenda bucal ampla, boca estendendo até os lados da cabeça, estômago de maior volume e com musculatura bastante elástica, intestino curto, reto e espesso, com maior número de dobras, são caraterísticas de peixes carnívoros. Segundo Silva et al. (22), conhecer a alimentação dos peixes proporciona informações sobre os aspectos básicos da sua biologia, como o crescimento, a adaptação, a maneira como exploram e utilizam os recursos disponíveis e as relações tróficas entre os organismos. A dieta da ictiofauna pode ser usada como uma ferramenta para a gestão de políticas públicas dos recursos hídricos e da fauna de peixes, podendo auxiliar na conservação desse grupo (23).

\section{CONCLUSÕES}

Os dados obtidos demonstram que o tamanho dos órgãos que compõe o tubo digestório da espécie $A$. lacustris não está relacionado ao tamanho do exemplar, pois nenhum órgão estudado apresentou a sequência decrescente estabelecida no comprimento padrão. A anatomia do tubo digestório da espécie estudada permite inferir seu hábito alimentar carnívoro. O estudo das características anatômicas do tubo digestório amplia o conhecimento biológico e ecológico da espécie $A$. lacustris e do grupo dos peixes. 
Conhecer a anatomia do tubo digestório permite verificar as relações entre as adaptações morfológicas e os hábitos alimentares e auxilia na compreensão do desempenho da espécie em ecossistemas naturais e na elaboração de propostas para a preservação ou manejo correto da espécie.

\section{REFERÊNCIAS}

1. Barcellos JFM, Branco E, Pontes D. Aspectos morfométricos do tubo digestório de Roeboides xenodon e Orthospinus franciscensis. Rev Biotemas. 2014;27(3):139-47. Doi: https://doi.org/10.5007/2175-7925.2014v27n3p139.

2. Rotta MA. Aspectos gerais da fisiologia e estrutura do sistema digestivo dos peixes relacionados à piscicultura. Corumbá: Embrapa Pantanal; 2003.

3. Joseph NS, Grande TC, Wilson MVH. Fishes of the world. 5a ed. New Jersey: John Wiley \& Sons; 2016.

4. Silva AT, Goitein R. Diet and feeding activity of Acestrorhynchus lacustris (Lütken, 1875) (Characiformes, Acestrorhynchidae) in the water reservoir at Ribeirão Claro, SP. Braz J Biol. 2009;69(3):757-62. doi: http://dx.doi.org/10.1590/S1519-69842009000400002.

5. Rocha AAF, Santos NCL, Pinto GA, Medeiros TN, Severi W. Diet composition and food overlap of Acestrorhynchus britskii and A. lacustris (Characiformes: Acestrorhynchidae) from Sobradinho reservoir, São Francisco river, Bahia State. Acta Sci Biol Sci. 2011;33(4):407-15. doi: https://doi.org/10.4025/actascibiolsci.v33i4.7240.

6. Teixeira I, Bennemann ST. Ecomorfologia refletindo a dieta dos peixes em um reservatório no sul do Brasil. Biota Neotrop. 2007;7(2):67-76. doi: https://doi.org/10.1590/S1676-06032007000200007.

7. Saccol-Pereira A, Milani PCC, Fialho CB. Primeiro registro de Acestrorhynchus pantaneiro Menezes, 1992 (Characiformes, Acestrorhynchidae) no sistema da laguna dos Patos, Rio Grande do Sul, Brasil. Biota Neotrop. 2006;6(3):1-4. doi: https://doi.org/10.1590/S1676-06032006000300017.

8. Fistarol PHB, Brandolff RS, Santos JYG. Análise fisiográfica da bacia do Rio de Ondas BA. In: Anais do 17o Simpósio Brasileiro de Sensoriamento Remoto - SBSR; 2015; João Pessoa. João Pessoa: INPE, SELPER; 2015. p. 5469-76.

9. Uieda VS, Castro RMC. Coleta e fixação de peixes de riachos. In: Caramaschi EP, Mazzoni R, Peres Neto PR. Ecologia de peixes de riachos. Rio de Janeiro: PPGE-UFRJ; 1999. v. 6, p. 1-22. (Série Oecologia Brasiliensis).

10. Vazzoler AEAM. Biologia da reprodução de peixes teleósteos: teoria e prática. Maringá: EDUEM, São Paulo: SBI; 1996.

11. Seixas Filho JT, Brás JM, Gomide ATM, Oliveira MGA, Donzele JL, Menin E. Anatomia funcional e morfometria dos intestinos e dos Cecos Pilóricos do Teleostei (Pisces) de Água Doce Piau (Leporinus friderici, Bloch, 1794). Rev Bras Zootec [Internet]. 2000 
[citado 18 Abr 2021];29(6):2181-92. Disponível em: http://www.sbz.org.br/revista/artigos/2723.pdf

12. Constantinescu GM, Habel RE, Sack WO, Schaller O, Simoes, P, Vos NR. Illustrated veterinary anatomical nomenclature. 2th ed. Germany: Enke; 1992.

13. Triola MF. Introdução à estatística. Rio de Janeiro: LTC; 2012.

14. Becker AG, Gonçalves JF, Garcia LO, Behr ER, Graça DL, Kurtz Filho M, et al. Morphometric parameters comparisons of the digestive tract of four teleosts with different feeding habits. Cienc Rural. 2010;40(4):862-6. doi: https://doi.org/10.1590/S010384782010005000049 .

15. Silva MT, Carneiro JH, Campos VPCC. Avaliação morfológica e morfométrica do sistema digestório do Bagre de Canal criado em cativeiro. Vet Zootec [Internet]. 2016 [citado 18 Abr 2021];23(1):105-13. Disponível em: https://www.bvsvet.org.br/vetindex/periodicos/veterinaria-e-zootecnia/23-(2016)-1/avaliacao-morfologicae-morfometrica-do-sistema-digestorio-do-bagre-de/

16. Britski HA, Sato Y, Rosa ABS. Manual de identificação de peixes da região de Três Marias: com chaves de identificação para os peixes da bacia do São Francisco. Brasília: CODEVASF; 1988.

17. Benedito E. Biologia e ecologia dos vertebrados. Rio de Janeiro: Roca; 2017.

18. Bellave SM, Pereira JGM, Schuingues CO, Lima MG, Costa GM. Descrição morfológica do estômago do barbado, Pinirampus pirinampu (Teleostei, Pimelodidae). Rev Cienc Agro-Ambient [Internet]. 2012 [citado 25 Abr 2021];10(2):213-8. Disponível em: http://www.unemat.br/revistas/rcaa/docs/vollo-

2/9_modelo_artigo_rcaa_v10n2a2012_gerlane.pdf

19. Liem KF, Bemis WE, Walker Jr WF, Grande L. Anatomia funcional dos vertebrados: uma perspectiva evolutiva. São Paulo: Cengage Learning; 2014.

20. Baldisserotto B. Fisiologia de peixes aplicada à piscicultura. Santa Maria: UFSM; 2002.

21. Amaral AA. Anatomia comparada do aparelho digestório de Acestrorhynchus britskii MENEZES, 1969 e Acestrorhynchus lacustris REINHARDT, 1874 (Pisces, Characidae, Acestrorhychinae). Rev Ceres [Internet]. 1990 [citado 27 Abr 2021];37(212):277-88. Disponível em: http://www.ceres.ufv.br/ojs/index.php/ceres/article/view/1981/24

22. Silva DA, Pessoa EKR, Gavilan SA, Costa L, Chellappa NT, Chellappa S. Ecologia alimentar de Astyanax lacustris (Osteichthyes: Characidae) na Lagoa do Piató, Assu, Rio Grande do Norte, Brasil. Biota Amazon. 2012;2(1):74-82. doi: http://dx.doi.org/10.18561/2179-5746/biotaamazonia.v2n2p54-61.

23. Lima FP, Nobile AB, Souza DF, Carvalho ED, Magnoni APV. Feeding ecology of Rhinodoras dorbignyi (Kner, 1855) (Siluriformes: Doradidae) in the Paranapanema River, SP, Brazil. Rev Biotemas. 2016;29(1):67-73. doi: https://doi.org/10.5007/21757925.2016v29n1p67. 
Recebido em: 29/07/2021

Aceito em: 23/11/2021

Guedes WF, Silva RM, Moreira MC, Pessoa LMB, Castro ERRS. Anatomia do tubo digestório da espécie Acestrorhynchus lacustris (Ostaryohisi, Characiformes) Lütken, 1875 do rio de Ondas, oeste da Bahia, Brasil. Vet. e Zootec. 2021; v28:001-015. 